\title{
Le rôle du système DOBIS à la Bibliothèque nationale du
} Canada

\section{The role of the DOBIS system at the National Library of Canada El papel del sistema DOBIS en la Biblioteca Nacional del Canadá}

\section{Louis J.S. Forget}

Volume 28, numéro 2, avril-juin 1982

Les réseaux documentaires au Québec

URI : https://id.erudit.org/iderudit/1053746ar

DOI : https://doi.org/10.7202/1053746ar

\section{Aller au sommaire du numéro}

\section{Éditeur(s)}

Association pour l'avancement des sciences et des techniques de la documentation (ASTED)

\section{ISSN}

\section{5-2340 (imprimé)}

2291-8949 (numérique)

\section{Découvrir la revue}

\section{Citer cet article}

Forget, L. J. (1982). Le rôle du système DOBIS à la Bibliothèque nationale du Canada. Documentation et bibliothèques, 28(2), 77-84.

https://doi.org/10.7202/1053746ar

\section{Résumé de l'article}

Cet article décrit le rôle du système DOBIS à la Bibliothèque nationale du Canada dans l'évolution du réseau d'information et de bibliothèques au Canada. DOBIS est un système intégré et en ligne de gestion de bibliothèque, développé par la Bibliothèque nationale du Canada et l'Institut canadien de l'information scientifique et technique, et exploité par la Bibliothèque nationale du Canada. Ce système est utilisé comme système/réseau partagé par la Bibliothèque nationale du Canada et l'Institut canadien de l'information scientifique et technique pour le soutien de leurs services nationaux, de même que par la Bibliothèque du Parlement et un nombre croissant de bibliothèques du gouvernement fédéral canadien pour le soutien de leurs opérations de bibliothèque. DOBIS est opérationnel depuis l'automne 1979. La base de données de DOBIS comprend maintenant plus de 2,3 millions de notices bibliographiques. Deux des principaux objectifs de la Bibliothèque nationale dans le développement du système DOBIS étaient de faciliter le partage des ressources de bibliothèque au Canada et de rendre les ressources d'information du pays plus facilement accessibles à tous les Canadiens. Afin de mieux poursuivre ces objectifs, la Bibliothèque nationale se propose d'utiliser le système DOBIS et sa base de données comme agents de sa participation au développement d'un réseau décentralisé d'information et de bibliothèques au Canada; réseau dont le but ultime est de procurer l'information voulue à qui la demande et au moment précis où elle est voulue. Les toutes dernières technologies de la télématique font parties intégrantes des travaux de ce développement. La stratégie fondamentale de ce développement vise à relier les systèmes autonomes actuels - DOBIS en est un exemple - de sorte que tous les utilisateurs d'un de ces systèmes puissent avoir accès aux services de tous les autres systèmes ainsi interreliés. Cette stratégie a été dénommée " interconnexion des systèmes ouverts ", parce qu'elle ouvre les systèmes et met leurs services individuels à la disposition de tous les autres systèmes faisant partie de l'interconnexion.
Tous droits réservés $₫$ Association pour l'avancement des sciences et des techniques de la documentation (ASTED), 1982
Ce document est protégé par la loi sur le droit d'auteur. L’utilisation des services d'Érudit (y compris la reproduction) est assujettie à sa politique d'utilisation que vous pouvez consulter en ligne.

https://apropos.erudit.org/fr/usagers/politique-dutilisation/ 


\section{Le rôle du système DOBIS à la Bibliothèque nationale du Canada}

Louis J.S. Forget*

Bibliothèque nationale du Canada

Ottawa

Cet article décrit le rôle du système DOBIS à la Bibliothèque nationale du Canada dans l'évolution du réseau d'information et de bibliothèques au Canada. DOBIS est un système intégré et en ligne de gestion de bibliothèque, développé par la Bibliothèque nationale du Canada et l'Institut canadien de l'information scientifique et technique, et exploité par la Bibliothèque nationale du Canada. Ce système est utilisé comme système/réseau partagé par la Bibliothèque nationale du Canada et l'Institut canadien de l'information scientifique et technique pour le soutien de leurs services nationaux, de même que par la Bibliothèque du Parlement et un nombre croissant de bibliothèques du gouvernement fédéral canadien pour le soutien de leurs opérations de bibliothèque. DOBIS est opérationnel depuis l'automne 1979. La base de données de DOBIS comprend maintenant plus de 2,3 millions de notices bibliographiques. Deux des principaux objectifs de la Bibliothèque nationale dans le développement du système DOBIS étaient de faciliter le partage des ressources de bibliothèque au Canada et de rendre les ressources d'information du pays plus facilement accessibles à tous les Canadiens. Afin de mieux poursuivre ces objectifs, la Bibliothèque nationale se propose d'utiliser le système DOBIS et sa base de données comme agents de sa participation au développement d'un réseau décentralisé d'information et de bibliothèques au Canada; réseau dont le but ultime est de procurer l'information voulue à qui la demande et au moment précis où elle est voulue. Les toutes dernières technologies de la télématique font parties intégrantes des travaux de ce développement. La stratégie fondamentale de ce développement vise à relier les systèmes autonomes actuels - DOBIS en est un exemple - de sorte que tous les utilisateurs d'un de ces systèmes puissent avoir accès aux services de tous les autres systèmes ainsi interreliés. Cette stratégie a été dénommée "interconnexion des systèmes ouverts", parce qu'elle ouvre les systèmes et met leurs services individuels à la disposition de tous les autres systèmes faisant partie de l'interconnexion.

\section{The role of the DOBIS system at the National Library of Canada}

This paper discusses the role of the DOBIS system at the National Library of Canada in the evolving library and information network in Canada. DOBIS is an on-line integrated library management system which has been developed by the National Library of Canada and the Canada Institute for Scientific and Technical Information, and is operated and maintained by the National Library of Canada. This system is used in a shared system/network mode by the National Library of Canada and the Canada Institute for Scientific and Technical Information to support their national services and also by the Library of Parliament and a growing number of Canadian federal government libraries to support their library operations. DOBIS has been operational since the fall of 1979. The DOBIS data base now contains over 2.3 million bibliographic records. Two of the major objectives of the National Library of Canada when it undertook the development of the DOBIS system were to facilitate library resources sharing across Canada, and to make the nation's information resources more readily accessible to all Canadians. In order to further fulfill these objectives, the National Library is now planning to use the DOBIS system and data base in its participation in the development of a decentralized Canadian library and information network, the goal of which is to get the right information where it is needed and when it is needed. The latest computer/ communications technologies are being employed in this development. The basic development strategy involves linking together existing independently operating systems, one example being DOBIS, so that users of one system can have access to the services of the other interconnected systems. This strategy is called "open systems interconnection", or OSI, because the services of all the interconnected systems are open to one another.

\section{El papel del sistema DOBIS en la Biblioteca Nacional del Canadá}

Este artículo describe el papel del sistema DOBIS en la Biblioteca Nacional del Canadá dentro de la evolución de las redes de información y de las bibliotecas en el Canadá. DOBIS es un sistema integrado por la línea directa de gestión de las bibliotecas, y ha sido desarrollado por la Biblioteca National del Canadá y el Instituto Canadiense de Información Cientifica y Técnica, pero es utilizado sólo por la Biblioteca

* L'auteur est directeur du Centre des systèmes de 
Nacional del Canadá. Este sistema se utiliza como un sistema-red, compartido tanto por la Biblioteca Nacional del Canadá como por el Instituto Canadiense de Información Científica y Técnica para sostener sus servicios nacionales. Es utilizado también por la Biblioteca del Parlamento y un número creciente de bibliotecas del gobierno federal canadiense para sostener sus operaciones de bibliotecas.

DOBIS está en actividad desde elotoño de 1979. La base de datos DOBIS comprende ahora más de 2,3 milliones de noticias bibliográficas. Dos de los principales objetivos de la Biblioteca Nacional, al empezar el desarrollo del sistema DOBIS, eran, por un lado, mejorar la distribución de los recursos de bibliotecas en el Canadá y por el otro, facilitar a todos los Canadienses el acceso a los recursos de información del pais. Para alcanzar aquellos objetivos, la Biblioteca Nacional se propone utilizar el sistema DOBIS y su base de datos como agentes de participación en el desarrollo de una red descentralizada de información $y$ de bibliotecas en el Canadá. Su objetivo principal es el de brindar al participante la información pedida precisamente en el momento que la quiere.

La tecnología más reciente está al servicio del desarrollo de DOBIS. La estrategia fundamental de este desarrollo aspira a poner en comunicación los sistemas autónomos actuales - DOBIS, por ejemplo de manera tal, que todos los usuarios de uno de aquellos sistemas puedan tener acceso a los servicios de los demás sistemas asi interconectados. Esta estrategia ha sido denominada "interconexión de sistemas abiertos", o bién OSI porque abre los sistemas y pone sus servicios individuales a la disposición de los demás sistemas que forman parte de la interconexión.

La Bibliothèque nationale du Canada (BNC) utilise l'informatique depuis quinze ans afin d'améliorer et de moderniser ses services et ainsi mieux jouer son rôle d'institution nationale. Après de très modestes débuts en 1967 avec l'automatisation de l'index de Canadiana, la bibliographie nationale, elle entreprit une étude globale sur la possibilité d'automatiser tous ses services. Le rapport final de cette étude ${ }^{1}$, remis au début de 1970, recommandait à la BNC d'implanter un système $d$ 'information intégré et automatisé, fondé sur le format MARC (Machine-Readable Cataloguing), lequel représentait déjà une norme pour l'échange de notices bibliographiques enregistrées sur bande magnétique. $A$ la suite de cette étude, la BNC a développé au début des années 1970 des systèmes automatisés exploités en traitement par lots pour son catalogage, y compris Canadiana, et ses fichiers d'autorité, en plus d'un service de distribution de notices MARC. Ces systèmes, ainsi que la planification du nouveau système DOBIS, ont été décrits en détail lors d'un colloque tenu en $1978^{2}$. Tous ces systèmes sont en train d'être transférés et intégrés au système DOBIS, objet de cet article.

1. Bibliothèque nationale du Canada, Un système unifié d'information pour la Bibliothèque nationale du Canada, résumé du rapport du projet d'analyse et d'automatisation des services, Ottawa, 1970.

2. Louis J.S. Forget, "Systèmes et réseaux à la Bibliothèque nationale du Canada; le rôle de la Bibliothèque nationale du Canada dans le réseau $d$ 'information et de bibliothèque au Canada/National Library of Canada systems and networks activities; the role of the National library of Canada in a Canada-wide network", in Systèmes et réseaux de bibliothèques/Libraries systems and networks. $4 e$ colloque conjoint ASTED/CLA, Québec, les 24 et 25 octobre 1978. Montréal, ASTED, 1979, p. 97-156.

\section{Les origines du système DOBIS}

Une analyse démontrait, dans le cadre de la planification de l'automatisation du Catalogue collectif canadien à la BNC en 1974, que la meilleure façon d'automatiser ce service était de le faire avec un système en ligne, qui pourrait par la suite servir de base à l'intégration de tous les autres systèmes de la BNC et même être utilisé comme système/réseau par l'ensemble des bibliothèques du gouvernement fédéral.

Après avoir examiné divers systèmes automatisés, la BNC concluait que des sommes considérables seraient épargnées si elle acquérait un système en ligne au lieu d'élaborer son propre système, en supposant que le système acquis réponde de façon satisfaisante à ses exigences. Elle décida donc à la fin de 1974 d'examiner les systèmes de bibliothèques susceptibles d'être utiles et de faire l'acquisition du plus pertinent durant l'année financière 1975-1976. Le système recherché devait satisfaire à quelques exigences essentielles: être en ligne; utiliser le concept de gestion d'une base de données stockant séparément les autorités et les descriptions bibliographiques, tout en établissant la liaison entre ces deux éléments; et comporter un certain nombre de modules pour servir de support à toutes les fonctions principales d'une bibliothèque. Des systèmes évalués, DOBIS (Dortmunder Bibliotheksystem), qu'élaborait I'Université de Dortmund en République Fédérale Allemande, se classait premier.

Ailleurs au Canada, d'autres groupes commençaient à s'intéresser au projet DOBIS et à suivre de près son élaboration. Un groupe de Canadiens 
visita I'Université de Dortmund en octobre 1975 pour faire une première évaluation de DOBIS et le reconnut comme l'un des meilleurs systèmes de gestion de bibliothèque disponibles. Afin d'épargner du temps et de l'argent dans l'application ultérieure de DOBIS au Canada, il fut décidé qu'une équipe de I'Université de Dortmund apporterait les modifications qui s'imposaient pour tenir compte du contexte canadien.

En décembre 1975, le gouvernement du Canada négociait des contrats avec I'Université de Dortmund afin d'acquérir les droits d'utilisation, de distribution et d'adaptation du système DOBIS au Canada. Les trois contrats furent signés à une date ultérieure. Toujours en décembre 1975, un groupe de travail formé de représentants des organismes canadiens qui avaient témoigné de l'intérêt pour le système DOBIS fut mis sur pied pour préparer la liste des premières modifications à y apporter. Ces demandes de modifications initiales furent envoyées à l'Université de Dortmund en janvier 1976. Elles portaient essentiellement sur l'utilisation du système par plusieurs institutions (réseau), le catalogage dans les deux langues officielles du Canada, et les normes du format MARC canadien.

En mars 1976, I'Institut canadien de l'information scientifique et technique (ICIST) et la BNC acceptaient de procéder conjointement à une évaluation globale du système DOBIS pour déterminer s'il pouvait répondre avantageusement aux besoins individuels et collectifs des deux institutions. De plus, le groupe TélécatUnicat des bibliothèques de I'Ontario et du Québec, par l'intermédiaire du Conseil des universités de l'Ontario, accepta de participer à l'évaluation.

La version modifiée de DOBIS, composée des modules de catalogage et de recherche, fut livrée à Ottawa le 24 mai 1976 et assemblée la semaine même. Les essais de réception des modifications apportées au système ont pris fin le 4 juin 1976. Le groupe de travail se montra satisfait de la quantité et de la qualité du travail accompli par l'équipe de conversion de DOBIS à I'Université de Dortmund, même si certaines des modifications demandées n'avaient pu être réalisées durant la brève période de conversion (du 1er janvier 1976 au 30 avril 1976).

Avant de poursuivre l'élaboration et la mise en application du logiciel de DOBIS, après sa livraison en mai 1976, il fut décidé d'évaluer ce système de façon rigoureuse. Cette évaluation se fit entre juin et décembre 1976 par une équipe de travail comprenant des membres de I'ICIST, de la BNC et du Conseil des universités de I'Ontario. Les objectifs du projet étaient d'étudier ce système tel qu'il nous avait été livré et d'en évaluer les capacités fonctionnelles, la performance et I'utilité.

L'évaluation ${ }^{3}$ permit de conclure en décembre 1976 que dans I'ensemble les aspects techniques et fonctionnels de DOBIS étaient satisfaisants mais qu'il y avait lieu d'améliorer certaines parties du système. En raison des améliorations qui s'imposaient, le système dut être considéré, pour l'instant, comme inadéquat dans la perspective d'une utilisation immédiate. Si le système, dans ses aspects techniques et fonctionnels, était conforme à nos attentes, il fallait toutefois le modifier et l'améliorer davantage pour qu'il puisse éventuellement répondre à toutes les exigences des bibliothèques participant à l'évaluation.

Puisque DOBIS constituait un fondement solide à toute expansion future, l'évaluation recommandait qu'il serve de base à la création d'un système/réseau de bibliothèques pour le gouvernement fédéral, pouvant se joindre à d'autres réseaux régionaux de bibliothèques au Canada. II était donc recommandé que soit entreprise la deuxième étape du Projet DOBIS avec les objectifs précis que voici:

- mettre en oeuvre les modifications requises pour porter le perfectionnement des modules de catalogage et de recherche au niveau fondamental, en vue de l'exploitation d'un projet pilote de base dans un contexte de production;

- poursuivre la mise au point, parallèlement à l'exploitation du projet pilote, des modules de catalogage et de recherche pour les porter au niveau optimal souhaité, en vue de les rendre aptes à une production intensive;

- étudier le développement des autres modules (acquisitions, contrôle des publications en série et prêt) et la possibilité de les intégrer au système DOBIS existant, selon l'ordre de priorité désiré.

Se fondant sur les résultats de l'évaluation de DOBIS et sur une étude menée en 1976 sur le rapport coûts/bénéfices d'utilisation de DOBIS, la BNC et I'ICIST ont poursuivi conjointement le développement de DOBIS en 1977 et son

3. Louis J.S. Forget et William L. Newman. "F.valuation of the DOBIS system for use in Canada", The Canadian Journal of Information Science/Revue canadienne des sciences de l'information, vol. 2 (May/mai 1977), 61-78. 
utilisation en projets pilotes en $1978^{4}$, pour ensuite le déclarer opérationnel en 1979 et l'étendre aux autres bibliothèques du gouvernement fédéral.

II est bon de noter aussi qu'en 1978 et 1979 I'Université de Louvain en Belgique s'est entendue avec I'Université de Dortmund pour développer les modules d'acquisitions et du prêt qui sont devenus le système LIBIS (Leuvens Integraal Bibliotheek System). En même temps, la compagnie IBM a obtenu les droits de commercialisation de tout le système DOBIS/LIBIS ${ }^{5,6}$. La Bibliothèque nationale du Canada a le droit de distribuer son logiciel DOBIS seulement au Canada. Ailleurs au Canada, le Centennial College de Toronto a adopté DOBIS pour le College Bibliocentre et l'a mis en opération au début de 1979. L'Université de l'Alberta commencera à utiliser DOBIS/LIBIS au cours de 1982 et d'autres institutions se montrent intéressées à ce système.

\section{Objectifs de DOBIS}

Le rapport sur l'avenir de la Bibliothèque nationale du Canada ${ }^{7}$ recommandait d'améliorer le partage de l'information et des ressources de bibliothèque au Canada par la création de réseaux parallèles décentralisés de collections et de bases de données bibliographiques. Ces réseaux sont perçus par la BNC comme des moyens de s'acquitter de son mandat qui est de faciliter I'utilisation des ressources de bibliothèque du pays par les citoyens du Canada et leur gouvernement. II en va de même pour l'utilisation du système DOBIS, développé et exploité en vue d'appuyer les programmes de la BNC et des autres institutions qui l'utilisent. On a donc essayé de définir les objectifs du système DOBIS comme suit:

1) servir de support efficace et dynamique à toutes les fonctions principales d'une bibliothèque afin d'en améliorer et d'en moderniser les services;

2) continuer à bâtir et à mettre à jour une base de données complète de notices bibliographiques pour le soutien des fonctions d'une bibliothèque;

4. "Rapport DOBIS et bibliographie", Nouvelles de la Bibliotheque nationale, vol. 10, no 6 (novembredécembre 1978), 1-6.

5. A. Stratton McAllister and Caryl McAllister, "A design for an on-line bibliographic database: the DOBIS/LIBIS database", Information Processing \& Management, vol. 17 (1981), 27-38.

6. Caryl McAllister and A. Stratton McAllister, "DOBIS/LIBIS: an integrated, on-line library management system", Journal of Library Automation, vol. 12 , no. 4 (December 1979), 300-313.

7. Bibliothèque nationale du Canada, L'avenir de la Bibliotheque nationale du Canada, Ottawa, 1979.
3) fournir des moyens rapides et précis d'identifier les ressources de bibliothèque et leurs localisations dans n'importe quelle bibliothèque du réseau canadien de bibliothèques;

4) réduire le taux d'accroissement des coûts de traitement et de recherche d'information;

5) fournir des renseignements statistiques qui pourraient servir par exemple à la rationalisation des collections au pays;

6) servir de système et de base de données partagés pour la BNC, I'ICIST et les autres bibliothèques du gouvernement fédéral tout en jouant le rôle de noeud à l'intérieur d'un réseau canadien décentralisé de bibliothèques avec des interfaces internationales.

\section{Caractéristiques de DOBIS}

Les fonctions et caractéristiques du système DOBIS à la BNC ont déjà fait l'objet d'un article dans une autre publication ${ }^{8}$. Voici néanmoins un sommaire de ces principales caractéristiques:

1) il s'agit d'un système intégré de gestion de bibliothèque comprenant des fonctions multiples telles que le catalogage, la recherche catalographique et documentaire, le catalogue collectif, les fichiers d'autorité, les acquisitions, le prêt et le contrôle des publications en série;

2) le système possède les propriétés d'un système de gestion de base de données, c'est-à-dire l'introduction et le stockage en un seul endroit des données permettant l'exploitation de multiples fonctions, ce qui réduit la répétition d'une même tâche et assure un meilleur contrôle bibliographique;

3) le système est en ligne ou en direct pour le soutien des fonctions précitées et la mise à jour se fait en temps réel, ce qui signifie que l'information est consignée dans la base de données et peut y être obtenue dès qu'elle $y$ est introduite;

4) le système possède de puissantes capacités de recherche en ligne, ce qui comprend la recherche libre, les termes de recherche flous, les termes de recherche tronqués, la recherche sur les noms (de personnes, de collectivités, de conférences), titres, matières, indices de classification, indices de placement,

8. William L. Newman et al., "DOBIS: the Canadian Government version", Canadian Library Journal, vol. 36, no. 4 (August 1979), 181-194. 
numéros de fiche de la Library of Congress, ISBN, ISSN, indices de bibliographie nationale, numéros de rapports techniques et autres, et enfin la recherche en direct sur mots clés en contexte dans les fichiers point d'accès de collectivités, conférences et titres;

5) le système comprend fichiers d'autorité et fichier topographique (propre à chacune des bibliothèques participantes) en ligne, et tous les autres outils de catalogage accessibles en un même endroit;

6) le système peut desservir simultanément plusieurs institutions car il contient des $\mathrm{fi}$ chiers généraux au niveau du système pour les données collectives et des fichiers facultatifs au niveau local pour les données particulières à chaque institution;

7) le système permet une accessibilité aux différentes fonctions protégée par un mot de passe et par des codes de sécurité contrôlés par la direction (un utilisateur doit être muni de l'autorisation nécessaire et détenir le code de sécurité requis concernant une fonction donnée pour exécuter cette fonction);

8) le système est bilingue, c'est-à-dire que l'utilisateur peut communiquer avec le système en français ou en anglais;

9) le système est compatible avec le MARC canadien et en plus il peut accepter des notices à divers degrés de qualité;

10) le système prévoit que le chargement de la base de données en différé peut s'effectuer simultanément avec la communication en direct;

11) le système permet le stockage, pour introduction implicite, de données particulières à chaque utilisateur;

12) le système facilite le dialogue au terminal par des répertoires des choix et commandes à utiliser et permet en plus le chainage des commandes pour les utilisateurs expérimentés;

13) le logiciel de DOBIS est rédigé en $P L / 1$ $(80 \%)$ et en assembleur $(20 \%)$;

14) le système est exploité sur ordinateur compatible avec le IBM 370 à partir de terminaux compatibles avec les IBM 3270 et utilise le système d'exploitation VS/1 et le moniteur de communications CICS/VS.
Le système DOBIS continue d'être modifié et amélioré afin de répondre aux nouveaux besoins des utilisateurs. Par exemple, on a implanté en 1981 la fusion automatique des notices sources chargées en différé dans la base de données DOBIS, pour que chaque ouvrage n'ait qu'une seule notice bibliographique et que tout changement apporté aux notices améliore la qualité générale de la base de données ${ }^{\circ}$.

\section{Situation actuelle}

Le système DOBIS, développé conjointement par la BNC et I'ICIST et exploité par la BNC, est opérationnel depuis l'automne 1979 sauf pour les modules des acquisitions, du prêt et du contrôle des publications en série. Ces trois derniers modules seront développés au cours des prochaines années. Le système de base maintenant opérationnel comprend les fonctions du catalogage, de la recherche catalographique et documentaire, du catalogue collectif et des fichiers d'autorité. La BNC et I'ICIST continuent d'améliorer ce système de base afin de répondre aux besoins de toutes les institutions qui l'utilisent. Ce système est utilisé comme système/réseau partagé par la BNC et I'ICIST pour le soutien de leurs services nationaux et aussi par la Bibliothèque du Parlement et un nombre croissant de bibliothèques du gouvernement fédéral canadien pour le soutien de leurs opérations de bibliothèque $10,11,12$.

La base de données de DOBIS comprend plus de 2300000 notices bibliographiques et s'enrichit d'environ 500000 nouvelles notices par année. Plus de 250 terminaux sont branchés sur DOBIS et on continuera d'en ajouter une soixantaine chaque année. Le système est exploité par un ordinateur IBM 370/3032 (8 méga-octets) localisé au Centre de calcul du Conseil national de recherches à Ottawa.

En plus d'avoir accès en ligne par terminaux à la base de données, les bibliothèques fédérales

9. Paul Mouland and Reg Webber, "Automated matching and amalgamation of MARC records in the DOBIS database/L'automatisation de la comparaison et de l'union des dossiers MARC dans la base de données DOBIS", The Canadian Journal of Information Science/Revue canadienne des sciences de l'information, vol. 6 (June/juin 1981), 57-65.

10. Mary Joan Dunn, "The Federal Government installation of DOBIS/Le gouvernement fédéral et l'installation du DOBIS", in Association des bibliothécaires du Québec/Quebec Library Association, Conférence annuelle/Annual Conference, Mirabel, Québec, 1-2 mai/May, 1981, Comptes rendus/ Proceedings, p. 41-54.

11. Mary Joan Dunn, "DOBIS in the Federal Government", BCLA Reporter, vol. 24, no. 2 (SeptemberOctober 1980), 2-6.

12. Mary Joan Dunn, "DOBIS", Argus, vol. 9, no 3 (mai-juin 1980), 105-109. 
qui utilisent le système DOBIS disposent d'une gamme de produits bibliographiques créés à partir de DOBIS: catalogues individuels ou collectifs sur microfiches, listes imprimées des publications en série et des acquisitions, notices lisibles par machine qui servent à alimenter d'autres systèmes, et de certains autres produits provisoires créés pour aider ces bibliothèques pendant la période de transition où leurs systèmes existants sont convertis au système DOBIS.

\section{DOBIS dans le réseau canadien}

On comprend de plus en plus qu'il est nécessaire et urgent pour le Canada de se doter d'un réseau automatisé d'information et de bibliothèques. Nous avons certains éléments de ce réseau déjà en place ${ }^{13}$ mais il reste encore beaucoup à faire pour la réalisation de ce grand projet. En même temps qu'elle commençait à planifier le développement d'un nouveau système en ligne en 1975, la BNC créa un Bureau des réseaux. Ce dernier était chargé d'élaborer des lignes de conduite et des plans pour le développement d'un réseau canadien d'information et de bibliothèques ainsi que d'étudier le rôle spécifique que devait y jouer la BNC.

Dans l'élaboration de ces lignes de conduite et de ces plans, le Bureau des réseaux a tenu compte des plans et des besoins des provinces et des régions, ainsi que du rapport final du Groupe de travail sur le Catalogue collectif canadien. II a aussi travaillé en étroite collaboration avec I'ICIST. De plus, entre 1976 et 1979, le Bureau a fait une vaste étude sur les centres bibliographiques automatisés du Canada. Cette étude avait pour but

- de cerner les principaux problèmes et défis se rattachant à la mise sur pied d'un réseau automatisé de bibliothèques au Canada, en donnant une vue d'ensemble des activités et des projets actuels des principaux centres bibliographiques automatisés existants et en voie de développement;

- de recommander les moyens permettant de promouvoir le développement souhaitable d'un réseau automatisé canadien de bibliothèques selon des méthodes de rentabilité et d'efficacité;

- de proposer des lignes de conduite favorisant le développement d'une base de données

13. Rosario de Varennes, "Un réseau informatique national de bibliothèques au Canada: réve ou réalité?/A Canadian national library and information network: dream or reality?", The Canadian Journal of Information Science/Revue canadienne des sciences de l'information, vol. 6 (June/juin 1981), 39-56. bibliographiques nationale fondée sur un réseau de centres bibliographiques automatisés nationaux et régionaux et leurs bases de données.

Cette étude a produit un document de base ${ }^{14}$ et un rapport final ${ }^{15}$ qui ont recommandé divers programmes en vue de l'établissement d'un réseau automatisé décentralisé de bibliothèques au Canada. L'un de ces programmes visait le développement et l'implantation du système DOBIS de la BNC comme système/réseau partagé par les bibliothèques du gouvernement fédéral et son interconnexion avec d'autres systèmes/réseaux d'information et de bibliothèques.

Peu de temps après avoir terminé cette étude, le Bureau des réseaux est devenu le Projet de réseau avec pour mission de conseiller la BNC sur l'établissement de programmes relatifs à l'organisation d'un réseau de bibliothèques au Canada. Le personnel affecté au Projet de réseau devait en outre veiller à ce que les bibliothèques, les consortiums et autres organismes participent à la planification et à la mise sur pied d'un réseau bibliographique national et décentralisé. Le Projet de réseau a développé le concept de la décentralisation $^{16}$ et a produit un document ${ }^{17}$ sur l'architecture de réseaux dans le contexte de l'interconnexion des systèmes ouverts qui sert de base aux développements en cours pour relier le système DOBIS de la BNC aux autres systèmes $^{18}$.

En 1981, le Projet de réseau de la BNC se transformait en Bureau du développement des réseaux. Ce nouveau Bureau est chargé de planifier et de coordonner la mise au point d'un réseau national de bibliothèques décentralisées dans le cadre de l'interconnexion des systèmes ouverts (Open Systems Interconnection/OSI) de l'Or-

14. R.M. Duchesne, M. Islam et D.A. Guenter, Elaboration d'un réseau informatique de bibliotheques au Canada: aperçu général, Ottawa, Bibliothèque nationale du Canada, 1979.

15. R.M. Duchesne, D.A. Guenter et M. Islam, Etablissement d'un réseau perfectionné de bibliothèques et de centres de documentation à l'échelle du Canada, Ottawa, Bibliotheque nationale du Canada, 1980.

16. Cynthia C. Durance and Mazharul Islam, "Decentralization vital to library networking", Computerworld, vol. 14, no. 48 (November 24, 1980), 7-8.

17. Edwin J. Buchinski et Mazharul Islam, Le contexte de l'interconnexion dans le cadre de l'élaboration d'un réseau bibliographique national, Ottawa, Bibliothèque nationale du Canada, 1980.

18. William L. Newman, "Open systems and DOBIS/ Systèmes ouverts et DOBIS", in Canadian Association for Information Science/Association canadienne des sciences de l'information, 9 th Annual Conference/9e conférence annuelle, Pointe-au-Pic, Québec, 27-30 May/mai, 1981, Proceedings/comptes rendus, vol. 9 (1981), $16 \mathrm{p}$. (disponible en accès direct chez QL Systems dans la base de données CAIS). 
ganisation internationale de normalisation ${ }^{19,20}$. L'interconnexion des systèmes ouverts suppose le recours à des procédures normalisées, permettant d'effectuer en direct l'échange d'information. Ces procédures visent à rendre possible aux bibliothèques déjà automatisées (ou qui le deviendront( l'échange malgré le fait qu'elles utilisent des ordinateurs disposant de matériels et de logiciels différents. Le point de départ de la BNC dans le développement de ce grand réseau automatisé est sa participation dans le projet iNET/ GATEWAY que le Groupe des communications informatiques du Réseau téléphonique transcanadien a mis récemment sur pied. Plusieurs groupes de services de toutes sortes participent à ce projet iNET qui s'inspire du modèle OSI et qui permettra de vérifier certains services de soutien facilitant l'accès à un réseau ouvert ${ }^{21}$. Un de ces groupes de services est le Groupe des services de bibliothèque qui réunit I'ICIST, I'Université du Québec, la Carleton University, I'University of Guelph, I'University of Waterloo et la BNC, laquelle fait fonction de coordonnateur de ce groupe.

L'objectif principal de ce groupe est de montrer comment la télématique peut faciliter l'accès aux ressources de bibliothèques et à l'information. Ainsi donc, les projets entrepris viennent appuyer les tâches fondamentales exécutées dans les bilbiothèques pour répondre aux besoins en information des clients (catalogage, diffusion de l'information et prêt entre bibliothèques) et favorisent l'expansion des services des bibliothèques participantes. Toutes les institutions membres du groupe accèdent ainsi à une gamme plus étendue de services d'information.

Les membres du Groupe des services de bibliothèque ont entrepris ensemble une série de projets. Deux de ces projets sont essentiels puisqu'ils facilitent aux membres l'accès au réseau: I'un d'eux relie chacun des participants à l'iNET/ GATEWAY par le biais du Datapac, leur permettant ainsi d'avoir accès facilement et rapidement aux bases de données et aux ordinateurs principaux des institutions membres, dont le système DOBIS de la BNC; l'autre porte sur la diffusion de répertoires iNET décrivant et commandant

19. Jerry Cashin, "Open system architecture: the ultimate window on the World?", Computerworld, vol. 16, no. 11a (March 17, 1982), 53-56.

20. Harold C. Folts, "Coming of age: a long-awaited standard for heterogeneous nets; open systems interconnection, a forthcoming international standard, is a solution to the problem of linking incompatible computers", Data Communications, vol. 10, no. 1 (January 1981), 63-73.

21. "Réseau intelligent de communications mis à l'essai". Informatique Québec, no 25 (février 1982), 1,15 .
I'accès aux diverses bases de données. D'autres projets étudient le partage des renseignements catalographiques réalisé par des recherches interactives en direct, cherchent à améliorer le prêt entre bibliothèques en ayant recours au courrier électronique, font en sorte que le vidéotex (Telidon) soit appliqué aux bibliothèques et vérifient l'accès aux bases de données d'autres groupes de services, par exemple celles des fournisseurs d'information.

La BNC espère ainsi que ce début d'interconnexion de son système DOBIS avec les systèmes des autres participants du projet iNET servira de base pour étendre cette interconnexion à partir de 1984 aux autres systèmes de bibliothèques au Canada et même à l'étranger afin de mieux jouer son rôle d'agence bibliographique nationale du Canada dans le cadre de grands programmes internationaux comme par exemple le Contrôle bibliographique universel (CBU) et la Disponibibité universelle des publications (DUP) de I'IFLA.

Le système DOBIS de la BNC joue déjà un rôle fondamental en ce sens qu'il sert de support à la BNC et à I'ICIST pour fournir leurs services nationaux en plus de servir de réseau aux bibliothèques du gouvernement fédéral qui l'utilisent. II est aussi l'outil qui permet à la BNC de participer au projet iNET. Si le projet iNET est un succès, le système/réseau DOBIS pourrait devenir un noeud vital dans un réseau d'information et de bibliothèques basé sur la télématique et dont les objectifs principaux seraient de faciliter davantage le partage des ressources de bibliothèque et de rendre les ressources $d$ 'information encore plus facilement accessibles à tous les Canadiens.

\section{Perspectives d'avenir}

Le rôle des bibliothèques dans la société des années 1980 est en pleine évolution ${ }^{22}$. Nous avons au Canada tous les outils et les éléments nécessaires à la réalisation du meilleur réseau automatisé d'information et de bibliothèques au monde. Plusieurs grandes bibliothèques et services d'information sont déjà automatisés au Canada. Nous avons facilement accès à toute la nouvelle technologie en informatique et en télécommunication et nous avons du personnel de plus en plus spécialisé et expérimenté en télématique. On peut donc envisager l'avenir avec beaucoup d'optimisme.

22. Maurice B. Line, "Libraries and information services in a post-technological society", Journal of Library Automation, vol. 14, no. 4 (December 1981), 252-267. 
Afin de mieux jouer son rôle dans le développement de ce réseau. la BNC s'est dotée du svstème DOBIS, un système moderne et flexible, qu'elle est en train de marier à la télématique pour mieux assumer à l'avenir son rôle de bibliothèque nationale et ainsi fournir à chaque Canadien les services qu'il attend de sa bibliothèque nationale.

Pour mieux planifier le rôle de la BNC dans les années 1980, le Conseil consultatif de la Bibliothèque nationale du Canada a créé en 1981 deux nouveaux comités dont le mandat est de promouvoir la mise sur pied de réseaux interdépendants et interreliés, visant à favoriser la mise en commun des ressources et des données bibliographiques au Canada. Ce sont le Comité du réseau de services bibliographiques et de communications, et le Comité du réseau de ressources. De plus, le Directeur général de la BNC a créé en 1980 un Groupe de travail sur les protocoles de communications/ ordinateurs pour l'échange de données bibliographiques. Ce groupe de travail est chargé d'identifier les protocoles (procédures normalisées) de télématiques dont I'application est particulière à l'échange de données bibliographiques et de recommander un protocole normalisé approprié à des applications bibliographiques particulières entre les systèmes de bibliothèques utilisant divers logiciels et matériels informatiques. Le travail de ce groupe est accompli dans le cadre du modèle d'interconnexion des systèmes ouverts. Ce groupe réunit des experts techniques spécialisés dans divers aspects de l'élaboration des systèmes bibliographiques, de l'élaboration des protocoles et des télécommunications. Les travaux de ces deux comités et de ce groupe de travail seront importants pour orienter les nouveaux développements du système DOBIS et les services qu'il assurera au sein du réseau décentralisé canadien d'information et de bibliothèques dans les années 1980.

II est à prévoir que l'interconnexion de DOBIS aux autres systèmes de bibliothèque et d'information ainsi que son intégration éventuelle à la bureautique, à la vidéographie et à toutes les autres technologies de l'information rapprocheront de plus en plus chaque Canadien des services de sa bibliothèque nationale. II est même possible que dans quelques années, les services de la BNC soient aussi près du citoyen canadien que les appareils de téléphone et de télévision dans son foyer, grâce aux développements du videotex et de la télématique.

\section{Conclusion}

Le système DOBIS à la BNC joue déjà un rôle important dans l'amélioration des services nationaux assurés par la BNC et I'ICIST. De plus, il sert de réseau aux autres bibliothèques du gouvernement fédéral qui l'utilisent. II aide aussi la BNC dans ses projets actuels basés sur la stratégie de l'interconnexion des systèmes ouverts qui devrait mener à la mise en place d'un véritable réseau automatisé et décentralisé d'information et de bibliothèques au Canada. Ainsi associé à la télématique dans un tel réseau, le système DOBIS de la BNC pourrait devenir un noeud vital de ce réseau qui faciliterait l'accès et le partage des ressources d'information et de bibliothèque au point $d$ 'en faire une sorte de bibliothèque illimitée ou une "bibliothèque virtuelle" ${ }^{23}$ pour chaque Canadien.

23. Michael Carmel, "Beyond networking", International Library Review, vol. 13, no. 3 (July 1981), 231-243. 